\title{
Rabaska
}

Revue d'ethnologie de l'Amérique française

\section{Laboratoire d'enquête ethnologique et de multimédia (Université Laval)}

\section{Laurier Turgeon}

Volume 17, 2019

URI : https://id.erudit.org/iderudit/1066053ar

DOI : https://doi.org/10.7202/1066053ar

Aller au sommaire du numéro

Éditeur(s)

Société québécoise d'ethnologie

ISSN

1703-7433 (imprimé)

1916-7350 (numérique)

Découvrir la revue

Citer ce document

Turgeon, L. (2019). Laboratoire d'enquête ethnologique et de multimédia

(Université Laval). Rabaska, 17, 392-395. https://doi.org/10.7202/1066053ar d'utilisation que vous pouvez consulter en ligne.

https://apropos.erudit.org/fr/usagers/politique-dutilisation/ 


\section{Célébration du $40^{e}$ anniversaire $d u C C Q$}

L'année 2019-2020 sera marquée par une série de petits événements et d'initiatives de communication pour marquer le $40^{\circ}$ anniversaire du CcQ, tous ayant comme objectif de mieux faire connaître l'ampleur et la valeur de l'expertise du personnel du CCQ. On veut ainsi démontrer comment le recours à cette mine d'expertise peut contribuer à mettre en valeur tous types de biens patrimoniaux au bénéfice de la population, des propriétaires et des communautés qui en ont la garde.

Ainsi, le CCQ :

- a participé aux assises annuelles de l'Union des municipalités du Québec afin d'y rencontrer des intervenants municipaux ayant la garde de biens patrimoniaux et de leur faire connaître les services que le CCQ peut leur rendre;

- a participé aux Rendez-vous Maestria, événement organisé par le Conseil des métiers d'art du Québec, afin de faire connaître les services du CCQ aux artisans et professionnels œuvrant dans le secteur du bâtiment et de la restauration architecturale; - a collaboré avec la Direction des communications et des affaires publiques du McC afin de réaliser une série de capsules vidéo présentant des réalisations marquantes du personnel du CCQ et pour augmenter la présence de l'organisation dans les médias sociaux par la publication de nouvelles brèves mises en lien avec le site Web du Centre;

- a travaillé en collaboration avec la Direction générale du patrimoine et les Directions régionales du Mcc dans la réalisation de leurs mandats respectifs en matière de conservation et de restauration d'œuvres et d'objets de patrimoine.

\section{Expertises sur des biens culturels}

La dernière année a aussi été remarquable par la réalisation d'un grand nombre d'expertises sur des biens religieux, des collections d'art public ou des éléments architecturaux. Ceci augure d'une prochaine année bien remplie au cours de laquelle l'expertise du personnel du CCQ continuera de rayonner et de rapporter des dividendes tangibles pour l'ensemble des citoyens et pour tous les propriétaires et gardiens de biens patrimoniaux.

PATRick Albert

\section{Laboratoire d'enquête ethnologique et de multimédia (LEEM)}

2256, Pavillon Charles-De-Koninck

Université Laval

1030, avenue des Sciences humaines

Québec (Québec)

G1V 0A6

Téléphone : (418) 656-2131,p. 403111 Courriel : Laurier.Turgeon@hst.ulaval.ca Toile : www.leem.ulaval.ca

L'année 2018-2019 fut pour la Laboratoire d'enquête ethnologique et de multimédia (LEEM) une année riche en projets et en expériences variées, tant en recherche fondamentale qu'en recherche appliquée. 
Publication d'un livre sur les relations entre Français et Amérindiens au XVI siècle Le volet de recherche fondamentale du Laboratoire a permis à Laurier Turgeon de mener à terme la rédaction et la publication d'un livre consacré aux relations entre Français et Amérindiens en Nouvelle-France au XVI ${ }^{\mathrm{e}}$ siècle. Laurier Turgeon a bénéficié d'une bourse de directeur d'études associé de la Fondation de la Maison des sciences de l'homme de Paris pendant le mois de décembre 2018 pour mener à terme les recherches et la rédaction du livre intitulé Une histoire de la Nouvelle-France : Français et Amérindiens au XVI siècle, paru chez Belin à Paris en mai 2019 et diffusé au Québec par Prologue. Ce livre envisage l'histoire du monde atlantique comme un lieu de contacts et d'échanges entre Européens et Amérindiens plutôt que comme un monde qui s'étend depuis un lieu central, l'Europe. L'étude des Français et des Amérindiens en Amérique du Nord au XVI siècle, jusqu'ici peu et mal connue, dévoile les origines de cette histoire croisée et connectée, voire interconnectée, en raison de la primauté et de l'importance de l'activité française dans cette partie du monde et de sa forte empreinte sur les sociétés amérindiennes. Le livre met l'accent sur l'étude de l'objet matériel parce c'est souvent par lui que la communication et l'échange interculturel se concrétisent. Bien avant de pratiquer l'échange linguistique, Français et Amérindiens ont échangé des objets. L'étude porte sur les usages français de deux objets d'origine américaine (la morue et les peaux de castor) et les appropriations amérindiennes de deux objets d'origine française (les chaudrons de cuivre et les perles de coquillage et de verre). Chaque objet offre une perspective différente sur cette dynamique de l'appropriation de la culture de l'autre, puis éclaire différents aspects de cette histoire interconnectée. L'analyse repose sur un vaste ensemble de sources - imprimées, manuscrites, iconographiques et archéologiques - largement inédites pour documenter cette histoire des objets.

C'est surtout le volet de recherche appliquée et de recherche création qui a retenu l'attention de l'équipe du LEEM, notamment les projets de l'interprétation en réalité augmentée de la Maison Longue de Wendake et d'interprétation multimédia du patrimoine maritime de la MRC de Lotbinière.

\section{Accroître l'interprétation de la Maison longue Ekionkiestha à Wendake}

Ce projet d'engagement partenarial financé par le Conseil de recherches en sciences humaines et sociales du Canada ( $\mathrm{CRSH}$ ) vise tout d'abord à répondre à une demande urgente du Musée huron-wendat de Wendake (Québec) de développement d'expertise dans l'utilisation de la réalité augmentée (RA). Une exposition sur les pratiques culturelles traditionnelles à l'époque du Sylvicole supérieur ( $\mathrm{XIV}^{\mathrm{e}}$ et $\mathrm{XV}^{\mathrm{e}}$ siècles) est en préparation pour la Maison longue Ekionkiestha. Son inauguration est prévue pour l'automne 2019 et le Musée souhaite y intégrer des composantes de RA. Pour y parvenir, nous avons mis sur pied un partenariat efficace reposant sur la mise en commun d'expertises complémentaires entre le Musée huron-wendat et le LeEm pour l'élaboration du programme d'interprétation : le Musée possède un important corpus de connaissances sur les modes de vie de cette époque et les objets pour les mettre en exposition ; le LeEM dispose d'une expertise théorique et méthodologique dans le domaine des médiations numériques des patrimoines et les équipements professionnels requis pour mettre en œuvre la $\mathrm{RA}$, acquis récemment à l'aide une subvention du fonds des leaders de la Fondation canadienne pour l'innovation. 
Le projet contribuera à l'avancement des connaissances dans le domaine des médiations numériques des patrimoines actuellement en pleine expansion. Si les technologies de la réalité augmentée sont aujourd'hui utilisées dans de nombreux domaines et champs d'expertises divers (industries automobile et aéronautique, cinéma, jeu vidéo, spectacle), elles tardent encore à faire leur entrée dans le champ du patrimoine en général et de l'interprétation du patrimoine autochtone en particulier. Elles génèrent des potentialités de médiation encore inexplorées que nous souhaitons justement appliquer au domaine du patrimoine, en recourant à des méthodes de médiation immersives et en facilitant leur réception, leur utilisation et leur appropriation, ce qui fait généralement défaut aux projets dans le domaine. Ces technologies peuvent être d'autant plus efficaces dans le cas de l'interprétation des cultures traditionnelles autochtones qu'elles permettent une expérience immersive dans un monde très différent du monde occidental d'aujourd'hui, et une meilleure connaissance de ces cultures peu et encore mal connues.

\section{Suivez L'Étoile - mise en valeur du patrimoine maritime en Lotbinière}

Le projet de documentation, de préservation et de mise en valeur de l'histoire et du patrimoine maritime de Lotbinière élaboré par la MrC de Lotbinière en collaboration avec le LEEM vise à réaliser 12 entrevues à caractère ethnologique avec des témoins de la vie maritime en Lotbinière, dans les quatre localités côtières de la MrC. Le LEEM effectue ces captations vidéo et participe à leur organisation sur les plans de la recherche et de la logistique. Les entrevues sont filmées sur le terrain, près du fleuve, ou dans un lieu adéquat où le participant est apte à livrer un témoignage complet, naturel et senti. Des images extérieures sont captées avec le témoin en marge de l'entrevue. Un guide d'écoute ethnologique est également élaboré pour chaque entretien. Le LEEM propose ensuite de réaliser 4 capsules vidéo de 3 à 4 minutes, à partir de ces 12 témoignages et d'images d'inserts illustrant la thématique et la région captées lors des entretiens. De plus, afin de valoriser davantage les porteurs du patrimoine et de la mémoire des quatre municipalités, le LEEM réalise 4 capsules supplémentaires, de 1 à 2 minutes, qui mettent en lumière des extraits des récits livrés par les intervenants. Ces capsules sont illustrées par des images d'archives, pour lesquelles le Laboratoire effectue la recherche, en collaboration avec les sociétés d'histoire du secteur.

\section{L'inventaire multimédia en ligne du patrimoine mobilier religieux de l'Île d'Orléans} Mettant à profit son expertise en inventorisation du patrimoine et ses équipements technologiques de pointe, le LEEM participe depuis 2017 à un projet qui vise à mieux connaître et à sauvegarder le patrimoine mobilier et immatériel religieux de l'Île d'Orléans. Le projet a permis d'inventorier encore cette année plusieurs centaines d'objets mobiliers dans les six églises de l'Île d'Orléans. Le projet permet de documenter une collection aujourd'hui méconnue et menacée malgré son ancienneté et sa richesse, puis de mettre en valeur les œuvres d'art, pièces d'orfèvrerie, meubles, documents et vêtements liturgiques présentant un intérêt historique, artistique ou ethnologique. Grâce à des enquêtes orales, il permet également de recueillir la mémoire vivante des Orléanais et de recenser des éléments du patrimoine immatériel, tels des 
savoir-faire liés aux usages des objets ou des pratiques culturelles et rituelles liées au culte. Afin de partager les connaissances et de sensibiliser le grand public au patrimoine religieux, les résultats de l'inventaire sont accessibles en ligne, notamment sur le Répertoire du patrimoine culturel du Québec. En plus de textes informatifs, les fiches d'inventaires présentent des contenus multimédias (photos 2D, photos 3D, vidéos) produits sur le terrain par les recherchistes associés au LeEm (Benoit Vaillancourt et Florence Gagnon-Brouillet). Réalisé sous les auspices du Comité du patrimoine religieux de l'Île d'Orléans (COPRIO), ce projet bénéficie du soutien financier du ministère de la Culture et des communications du Québec, du Diocèse de Québec, des fabriques de Sainte-Trinité-d'Orléans et de la Sainte-Famille-d'Orléans.

\section{Pour une nouvelle gouvernance des musées : enjeux et perspectives}

Laurier Turgeon lance cette année un nouveau projet triennal sur la gouvernance des musées, avec Yves Bergeron de l'UQÀm, et Habib Saïdi et Jean-François Gauvin de l'Université Laval, généreusement financé par le Conseil de recherches en sciences humaines du Canada (CRSH 2019-2022). Ce sujet est devenu aujourd'hui un enjeu majeur aussi bien dans les recherches scientifiques que pour les politiques culturelles et patrimoniales. Les musées vivent des transformations structurales importantes caractérisées par la présence de plus en plus marquée d'institutions ou de collections muséales parapubliques ou privées, la diversification des modalités d'accès au musée, l'approche touristique de la visite, la prédominance du numérique dans la mise en exposition des objets et dans la communication avec les visiteurs. À cette liste déjà longue s'ajoute la tendance forte des chercheurs et des visiteurs à remettre en cause le pouvoir de l'État et des organisations internationales dans la gestion du patrimoine afin de favoriser plutôt des modes de gestion communautaires et participatifs où le musée sert de « zone de contact » entre les différents acteurs, porteurs, transmetteurs et récepteurs de ce patrimoine. La gouvernance des musées représente également un domaine de recherche intersectoriel, au carrefour des préoccupations de la gestion et du patrimoine, de la démocratie participative et de la culture publique, croisant souvent plusieurs champs d'intérêts d'institutions culturelles internationales, nationales, territoriales et associatives. Les enquêtes, menées par l'équipe des quatre professeurs et des quatre étudiants aux cycles supérieurs, porteront sur une dizaine de musées au Canada, aux États-Unis et en Europe, dont le Musée de la civilisation à Québec, le Musée de la mémoire vivante à Saint-Jean-Port-Joli, le Musée des beaux-arts de Montréal, le Musée des sciences et des technologies du Canada, le Musée des droits de la personne du Canada, le Musée d'anthropologie de l'Université de la ColombieBritannique, le Smithsonian Institution à Washington, le Tate Museum à Londres, le Musée des Arts et métiers de Paris et le Musée d'ethnographie de Neuchâtel.

LAURIER TURgEON 